\title{
HSD3B1 genotype identifies glucocorticoid responsiveness in severe asthma
}

\author{
Joe Zein ${ }^{a}$, Benjamin Gaston ${ }^{b}$, Peter Bazeley ${ }^{a}$, Mark D. DeBoer ${ }^{c}$, Robert P. Igo Jr. ${ }^{d}$, Eugene R. Bleecker ${ }^{e}$, Deborah Meyers ${ }^{e}$, \\ Suzy Comhair ${ }^{a}$, Nadzeya V. Marozkina ${ }^{f} \odot$, Calvin Cotton ${ }^{f}$, Mona Patel ${ }^{a}$, Mohammad Alyamani ${ }^{a}$, Weiling $\mathbf{X u}^{a}$, \\ William W. Busse ${ }^{\mathrm{g}}$, William J. Calhoun ${ }^{\mathrm{h}}$, Victor Ortegai, Gregory A. Hawkins', Mario Castroj, Kian Fan Chung ${ }^{\mathrm{k}}\left({ }^{\mathrm{D}}\right.$, \\ John V. Fahy', Anne M. Fitzpatrick ${ }^{m}$, Elliot Israel ${ }^{n}$, Nizar N. Jarjour ${ }^{9}$, Bruce Levy ${ }^{n}$, David T. Mauger ${ }^{\circ}$, Wendy C. Moore', \\ Patricia Noel $^{p}$, Stephen P. Petersi', W. Gerald Teague ${ }^{c}$, Sally E. Wenzel ${ }^{q}$, Serpil C. Erzurum ${ }^{a}$, and Nima Sharifi ${ }^{a, 1}{ }^{1}$
}

aLerner Research Institute and the Respiratory Institute, Cleveland Clinic, Cleveland, OH 44195; ${ }^{b}$ Herman Wells Center for Pediatric Research, Indiana University School of Medicine, Indianapolis, IN 46202; 'Department of Pediatrics, University of Virginia, Charlottesville, VA 22904; ${ }^{\mathrm{d} D e p a r t m e n t}$ of Population and Quantitative Health Sciences, Case Western Reserve University, Cleveland, OH 44106; ${ }^{\circ}$ Department of Medicine, University of Arizona Health Sciences, Tucson, AZ 85721; ' Department of Pediatrics, Rainbow Babies and Children's Hospital, and Case Western Reserve University, Cleveland, $\mathrm{OH} 44106 ;{ }^{9}$ Department of Medicine, University of Wisconsin School of Medicine and Public Health, Madison, WI 53706; ${ }^{\mathrm{h}}$ Department of Medicine, University of Texas Medical Branch, TX 77555; 'Internal Medicine, Wake Forest University School of Medicine, Winston-Salem, NC 27587; 'Department of Medicine, University of Kansas School of Medicine, Kansas City, KS 66160; 'The National Heart \& Lung Institute, Imperial College London, London SW7 2AZ, United Kingdom; 'Department of Pediatrics, San Francisco School of Medicine, University of California, San Francisco, CA 94143; ${ }^{\mathrm{m} D e p a r t m e n t}$ of Pediatrics, Emory University School of Medicine, Atlanta, GA 30322; " ${ }^{\circ}$ epartment of Medicine, Harvard Medical School, Boston, MA 02115; ${ }^{\circ}$ Center for Biostatistics and Epidemiology, Pennsylvania

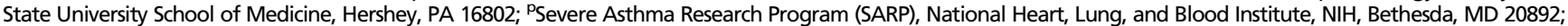
and 'University of Pittsburgh Asthma Institute, University of Pittsburgh Medical Center-University of Pittsburgh School of Medicine, Pittsburgh, PA 15261

Edited by David J. Mangelsdorf, The University of Texas Southwestern Medical Center, Dallas, TX, and approved December 2, 2019 (received for review October 31, 2019)

Asthma resistance to glucocorticoid treatment is a major health problem with unclear etiology. Glucocorticoids inhibit adrenal androgen production. However, androgens have potential benefits in

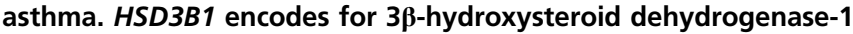

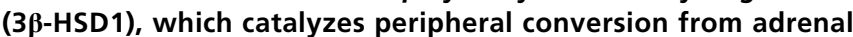
dehydroepiandrosterone (DHEA) to potent androgens and has a germline missense-encoding polymorphism. The adrenal restrictive HSD3B1(1245A) allele limits conversion, whereas the adrenal permissive HSD3B1(1245C) allele increases DHEA metabolism to potent androgens. In the Severe Asthma Research Program (SARP) III cohort, we determined the association between DHEA-sulfate and percentage predicted forced expiratory volume in $1 \mathrm{~s}\left(\mathrm{FEV}_{\mathbf{1}} \mathrm{PP}\right)$. HSD3B1(1245) genotypes were assessed, and association between adrenal restrictive and adrenal permissive alleles and $\mathrm{FEV}_{\mathbf{1}} \mathrm{PP}$ in patients with (GC) and without (noGC) daily oral glucocorticoid treatment was determined $(n=318)$. Validation was performed in a second cohort (SARP I\&ll; $\boldsymbol{n}=184$ ). DHEA-sulfate is associated with $\mathrm{FEV}_{\mathbf{1}} \mathrm{PP}$ and is suppressed with GC treatment. GC patients homozygous for the adrenal restrictive genotype have lower FEV 1 PP compared with noGC patients $(54.3 \%$ vs. $75.1 \%$; $P<0.001)$. In patients with the homozygous adrenal permissive genotype, there was no FEV ${ }_{1}$ PP difference in GC vs. noGC patients $(73.4 \%$ vs. $78.9 \% ; P=$ 0.39). Results were independently confirmed: $F V_{1} P P$ for homozygous adrenal restrictive genotype in GC vs. noGC is 49.8 vs. $63.4(P<0.001)$, and for homozygous adrenal permissive genotype, it is 66.7 vs. 67.7 $(P=0.92)$. The adrenal restrictive HSD3B1(1245) genotype is associated with GC resistance. This effect appears to be driven by GC sup-

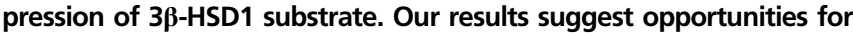
prediction of GC resistance and pharmacologic intervention.

steroids | glucocorticoids | inflammation | androgens | HSD3B1

S ince their discovery and introduction into clinical medicine about 70 y ago, glucocorticoids (GCs) have been recognized as eliciting a systemic antiinflammatory response, and currently play a major role in the treatment of severe asthma and other inflammatory disease processes (1). However, unresponsiveness to GC treatment is a major barrier to treatment of inflammatory disease processes, and the underlying mechanisms of this clinical entity have yet to be clearly elucidated (2). Management of GCunresponsive disease represents a significant challenge for the treatment of asthma. Indeed, severe asthma is generally defined as asthma that remains symptomatic despite high-dose inhaled GC and/or systemic GC therapy (3).
Suppression of endogenous adrenal androgens and cortisol is a known consequence of systemic GC treatment. Substantial evidence suggests that stimulation of the androgen receptor (AR) expressed in the lung could be beneficial in asthma. Androgens inhibit human airway smooth muscle and fibroblast proliferation (4-6), promote airway smooth muscle relaxation (7), and inhibit both Th2 and Th1 inflammation in animal models of asthma (8, 9). Androgens are associated with better lung function in large healthy cohorts $(10,11)$ and in asthma (12). Increasing circulating

\section{Significance}

Although resistance to glucocorticoids is a major clinical problem, the underlying mechanisms are unknown. It is known that glucocorticoid use can suppress adrenal androgen production. In population studies, animal models, and cell culture experiments, androgens are associated with several benefits in asthma, but neither androgen use in glucocorticoid-resistant asthma nor the genetic determinants of androgen responsiveness have been studied in humans. A missense-encoding variant in HSD3B1 is known to regulate conversion from adrenal precursors to potent androgens and clinical outcomes in prostate cancer. This is the first genetic evidence to our knowledge that implicates an androgen synthesis variant in resistance to glucocorticoids for asthma or any other inflammatory disease. Furthermore, this study demonstrates an adverse consequence of adrenal androgen suppression with glucocorticoid therapy.

Author contributions: J.Z., B.G., M.D.D., E.R.B., D. Myers, S.C., W.W.B., W.J.C., V.O., G.A.H., M.C., K.F.C., J.V.F., A.M.F., E.I., N.N.J., B.L., D.T.M., W. C.M., P.N., S.P.P., W.G.T., S.E.W., S.C.E., and N.S. designed research; J.Z., B.G., P.B., M.D.D., S.C., N.V.M., C.C., M.P., M.A., W.X., W.W.B., S.C.E., and N.S. performed research; J.Z., W.J.C., V.O., G.A.H., M.C., K.F.C., J.V.F., A.M.F., E.I., N.N.J., B.L., D.T.M., W.C.M., S.P.P., W.G.T., S.E.W., S.C.E., and N.S. contributed new reagents/analytic tools; J.Z., B.G., P.B., M.D.D., R.P.I., E.R.B., D. Myers, S.C., N.V.M., M.P., M.A., W.W.B., W.J.C., V.O., G.A.H., M.C., K.F.C., J.V.F., A.M.F., E.I., N.N.J., B.L., D.T.M., W.C.M., S.P.P., W.G.T., S.E.W., S.C.E., and N.S. analyzed data; and J.Z., B.G., S.C.E., and N.S. wrote the paper.

Competing interest statement: Cleveland Clinic has applied for patents on HSD3B1.

This article is a PNAS Direct Submission.

This open access article is distributed under Creative Commons Attribution-NonCommercialNoDerivatives License 4.0 (CC BY-NC-ND).

${ }^{1}$ To whom correspondence may be addressed. Email: sharifn@ccf.org.

This article contains supporting information online at https://www.pnas.org/lookup/suppl/ doi:10.1073/pnas.1918819117/-/DCSupplemental.

First published January 13, 2020. 
levels of adrenal and gonadal androgens in males and females during adolescence are associated with improving asthma during adolescence (12). However, the role of GC-induced androgen suppression in the pathophysiology of severe, GC-resistant human asthma is not established.

The androgen dehydroepiandrosterone (DHEA) and its sulfate (DHEA-S) are secreted from the adrenal reticularis $(13,14)$, and together are the most abundant steroid in circulation. However, its function is not known. DHEA-S may have an immunomodulatory effect, but clinical results have been inconsistent, possibly because variations in DHEA-S metabolism are generally not taken into account (15). In peripheral tissues, DHEA is metabolized by the

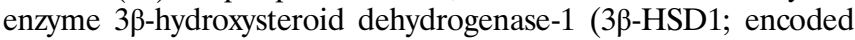
by $H S D 3 B 1$ ) to potent downstream androgens (e.g., testosterone and dihydrotestosterone). A common missense-encoding variant in $H S D 3 B 1$, rs 1047303 (c.1245C > A, p.T367N), regulates biochemical function and clinical phenotypes. The HSD3B1(1245A) allele encodes for an adrenal restrictive enzyme that limits conversion from DHEA to downstream androgens, whereas the $H S D 3 B 1(1245 C)$ allele encodes for an adrenal permissive enzyme that results in increased metabolic flux from DHEA to more potent androgens (16). Multiple studies from the United States, Japan, and Spain, now further confirmed in a phase 3 clinical trial, clearly show that men with advanced prostate cancer treated with castration who inherit the adrenal permissive $3 \beta$ HSD1 enzyme, which confers more rapid conversion from DHEA to potent androgens, have more rapid onset of androgendriven disease progression (17-22). These studies establish that clear clinical phenotypes are associated with $H S D 3 B 1$ genotypes that biochemically confer fast and slow metabolic flux to potent androgens. We hypothesized that the restrictive HSD3B1 genotype that disables DHEA-S conversion to potent androgens impairs $\mathrm{FEV}_{1} \mathrm{PP}$ specifically when GC treatment suppresses adrenal DHEA-S production, limiting substrate availability for $3 \beta$-HSD1 and possibly providing a mechanistic explanation for GC-resistant severe asthma in patients with this genotype.

Severe Asthma Research Programs (SARP) are comprehensive networks sponsored by the National Institutes of Health/ National Heart, Lung, and Blood Institute that aimed to characterize and understand the pathobiology of severe asthma. Here, we determined $\mathrm{FEV}_{1} \mathrm{PP}$ in the SARP III cohort for patients with asthma treated with (GC) and without (noGC) daily oral GCs. Confirmatory studies were performed in patients with severe asthma from the SARP I\&II study.

\section{Methods}

Subjects. Individuals included in this analysis comprised a subset of 318 Caucasian (from 488 patients of all races) adults (aged $>18 \mathrm{y}$ ) with asthma enrolled in the SARP III study for whom genotyping data and pulmonary function testing were available. We only included Caucasian patients in our study because of the numbers of patients available for analysis and the higher adrenal permissive HSD3B1(1245C) allele frequency of $30 \%$ to $40 \%$ in Caucasians, as opposed to $\sim 8 \%$ in people of African descent.

SARP III, a network of 11 clinical research centers across the United States, recruited 713 participants with asthma between 1 November 2012, and 1 October 2015. Of those 713 initially recruited participants with asthma, 526 were adults (aged $>18 \mathrm{y}$ ), $60 \%$ had severe asthma as defined by the European Respiratory Society/American Thoracic Society criteria (3), and 17\% ( $21 \%$ of adult Caucasians) were chronically treated with chronic oral and/or injectable GCs (i.e., systemic). The remaining $40 \%$ of patients with asthma did not meet the criteria for severe asthma and were classified as having nonsevere asthma. Among the $89(17 \%)$ adult subjects treated with systemic GCs in SARP III, 55 received daily oral GCs only, 15 received daily oral GCS with additional GC injections, and 19 received intermittent GC injections alone. In the group that received intermittent GC injections alone, the majority (14 participants) received 1 to 3 injections per year. The rest ( 5 subjects) received more than 6 injections per year. While the suppressive effect of GCs is predictable with chronic daily GC use, intermittent GC injections might have an inconsistent effect on adrenal suppression, and accordingly, participants receiving such therapy were excluded from the analysis. Patients were also excluded if they were pregnant or breast-feeding during the initial characterization period, had a history of premature birth ( $<35 \mathrm{wk}$ gestation), or had a diagnosis of any other chronic pulmonary disorder. All patients were not active smokers (past smokers should not have smoked within a year or exceeded 10 pack-years of tobacco use if $>30$ y of age, or $<5$ pack-years if $<30 \mathrm{y}$ of age) and were required to have evidence of bronchial hyperresponsiveness (defined as a PC20 methacholine value $<16 \mathrm{mg} / \mathrm{mL}$ ) or reversible airflow obstruction, as evidenced by an increase in $\mathrm{FEV}_{1}$ of $12 \%$ or greater after albuterol inhalation, ipratropium bromide inhalation, or both. Spirometry was performed according to the American Thoracic Society/European Respiratory Society guidelines (23, 24). The 2012 Global Lung Initiative standard reference equations were used to predict spirometric reference values (25). DHEA-S levels were analyzed at the University of Virginia Center for Research in Reproduction Ligand Core Laboratory, using the Siemens Immulite 2000 immunoassay system, which has a lower limit of detection for DHEA-S of $15 \mu \mathrm{g} / \mathrm{dL}$ Information on SARP III network, protocol, and characterization procedures have been published previously (26-28). All participants provided written informed consent. The institutional review board at each center approved the study. The study is listed on ClinicalTrials.gov.

To replicate primary findings, 184 Caucasian participants (from a total of 263 of all races) with severe asthma were selected from the SARP I\&II cohorts. Similarly, SARP I\&II is a National Institutes of Health/National Heart, Lung, and Blood Institute-sponsored multicenter study that recruited patients with asthma between 2001 and 2012 from 9 sites in the United States and 1 in the United Kingdom. However, as compared with SARP III, patients with asthma enrolled in SARP I\&II were less likely to have severe asthma ( $40 \%$ vs. $60 \%)$ (28-30). In contrast to SARP III, severe asthma was defined in SARP I\&II according to the initial ATS workshop definition of severe asthma (31).

Statistical Analysis. Whole-genome sequence of 1,888 patients enrolled in SARP I, II, and III was released by the Trans-Omics for Precision Medicine (TOPMed) program (https://www.nhlbiwgs.org/) in its genotype call sets freeze 6 a version. Whole-genome sequence with a read depth of $38 \times$ was performed on blood DNA, using Illumina HiSeq $X$ technology. The TOPMed freeze 6 a genotype call set includes 107,047 samples and $642 \mathrm{M}$ high-quality variants genomewide, $>7.6 \mathrm{M}$ coding variants, and $>350,000$ loss-of-function variants. Genotype calling and quality control were performed by the Informatics Research Center, led by the University of Michigan.

Genotypes for variant rs1047303 (position chr1:119514623 [build GRCh38.p12]) in SARP I, II, and III were extracted with PLINK2 $(32,33)$ (https:// www.cog-genomics.org/plink2). HSD3B1 genotypes were directly confirmed in 28 patients, using a method previously validated with $100 \%$ match (17).

Student's $t$ test was used for 2 group comparisons of continuous normally distributed variables. The means of the 3 different genotype groups were compared using the ANOVA test. Pairwise comparisons were performed using the Tukey-Kramer Honest Significant Differences (HSD) test. Other wise, Wilcoxon's rank sum test or Kruskal-Wallis one-way ANOVA were used when normality assumptions were not met. Categorical variables were compared using a $\chi^{2}$ test. We assumed an additive model of inheritance, in which predictor variables were an additive effect for the rs1047303 genotype (coded as the number of $\mathrm{C}$ alleles). Interaction terms were included between rs1047303 genetic variants and daily oral GC as the dependent variables of the prebronchodilator FEV ${ }_{1} \mathrm{PP}$ (pre-FEV ${ }_{1} \mathrm{PP}$ ) post-FEV ${ }_{1} \mathrm{PP}$. Models were fit under the assumption of a normal distribution for FEV ${ }_{1} P P$. All statistical analyses were conducted with $R$, version 3.5.3 (R Project for Statistical Computing, Vienna, Austria). A $P$ value $<0.05$ was considered statistically significant because only one position was tested.

Data Availability. TOPMed genomic data and preexisting parent study phenotypic data are made available to the scientific community in study-specific accessions in the database of Genotypes and Phenotypes (https:// www.ncbi.nlm.nih.gov/gap/).

\section{Results}

Baseline Characteristics. Baseline characteristics of Caucasian SARP III participants with asthma and SARP I\&II participants with severe asthma are listed in Table 1 . Aside from higher body mass index (BMI) in participants with the $\mathrm{CC}$ compared with the AA genotype enrolled in SARP III, patients with the AA, AC, and $\mathrm{CC}$ genotypes had similar baseline characteristics. Other than the $\mathrm{CC}$ genotype being associated with higher $\mathrm{FEV}_{1} / \mathrm{FVC}$ ratio compared with $\mathrm{AA}$ and $\mathrm{AC}$, all other characteristics were not significantly different in participants with severe asthma enrolled in SARP I, II, or III. 
Table 1. Baseline characteristics comparing the 2 SARP cohorts, SARP III vs. SARP I\&Il, stratified by HSD3B1(1245) genotypes*

\begin{tabular}{|c|c|c|c|c|c|c|c|c|}
\hline & \multicolumn{4}{|c|}{ SARP III } & \multicolumn{4}{|c|}{ SARP I\& II } \\
\hline & AA & $A C$ & CC & $P$ value & AA & $A C$ & $\mathrm{CC}$ & $P$ value \\
\hline$N$ & 146 & 131 & 41 & & 80 & 79 & 25 & \\
\hline Female sex, $n(\%)$ & $92(63.0)$ & $80(61.1)$ & $31(75.6)$ & 0.230 & $46(57.5)$ & $47(59.5)$ & $20(80.0)$ & 0.11 \\
\hline $\mathrm{BMI}^{\dagger}$ & $30.2 \pm 6.2$ & $31.8 \pm 8.6$ & $33.4 \pm 9.8$ & 0.038 & $30.3 \pm 7.2$ & $30.5 \pm 7.5$ & $29.9 \pm 6.9$ & 0.93 \\
\hline Daily oral GC therapy, $n(\%)$ & $23(15.8)$ & $24(18.3)$ & $8(19.5)$ & 0.787 & $35(43.8)$ & $30(38.0)$ & $10(40.0)$ & 0.76 \\
\hline Pre-FEV1, $\%$ of predicted value & $71.8 \pm 19.3$ & $72.6 \pm 21.8$ & $77.9 \pm 16.3$ & 0.224 & $57.4 \pm 19.2$ & $61.1 \pm 21.6$ & $67.3 \pm 23.7$ & 0.09 \\
\hline Post-FEV1, $\%$ of predicted value & $80.8 \pm 20.2$ & $80.3 \pm 20.8$ & $88.0 \pm 16.5$ & 0.083 & $73.5 \pm 19.2$ & $76.3 \pm 20.7$ & $79.3 \pm 24.8$ & 0.43 \\
\hline
\end{tabular}

GC, daily oral glucocorticoid therapy; FVC, forced vital capacity; Post-, postbronchodilator; Pre-, prebronchodilator.

*Plus-minus values are means \pm SD.

${ }^{\dagger}$ The $\mathrm{BMI}$ is the weight in kilograms divided by the square of the height in meters.

${ }^{\ddagger}$ Analyzed SARP I\&ll cohort includes patients with severe asthma.

Baseline DHEA-S and FEV $\mathbf{1}$ PP. FEV ${ }_{1} \mathrm{PP}$ was weakly associated with serum DHEA-S in both men and women. In the SARP III cohort, the $R^{2}$ (proportion of $\mathrm{FEV}_{1} \mathrm{PP}$ variability explained by DHEA-S) was 0.04 for all races $(n=314 ; P<0.001)$ and 0.04 $(n=203 ; P<0.001)$ for Caucasians. Similarly, for severe asthma in SARP I\&II, the $R^{2}$ was $0.13(n=271 ; P<0.001)$ for all races and $0.20(n=178 ; P<0.001)$ in Caucasians (Fig. $1 A$ and $S I$ Appendix, Table S1). This association appears to be driven by patients with low DHEA-S (i.e., first quartile; SI Appendix, Fig. S1). Strikingly, no women and very few men with DHEA-S levels over $200 \mu \mathrm{g} / \mathrm{dL}$ in either cohort had a baseline $\mathrm{FEV}_{1} \mathrm{PP}$ of less than $75 \%$ in SARP III.

DHEA-S Suppression Is Associated with Oral GC Use. Daily oral GC therapy was commonly used in SARP III with median dose and duration that did not differ among the $3 H S D 3 B 1$ genotypes. The median duration was 12 mo for each of the 3 genotypes $(P=0.18$ by Kruskal-Wallis test), and the median dose was $10 \mathrm{mg}$ prednisone ( $P=0.74$ by Kruskal-Wallis test). Overall, $22.6 \%$ (29\% of Caucasians) of adults with severe asthma enrolled in SARP III were treated with daily oral GC therapy (26) (SI Appendix, Table S2). Endogenous circulating cortisol declined significantly $(P<$ 0.001 ) in patients receiving oral GCs, confirming both patient compliance and adrenal suppression (SI Appendix, Fig. S2). As expected, our analysis of DHEA-S from 314 adult participants with asthma enrolled in SARP III showed significantly lower plasma DHEA-S levels in patients treated with daily oral GC therapy, as opposed to those not receiving daily oral GCs for both men and women. Not surprisingly, DHEA-S decline occurs in men and women (Fig. 1B), irrespective of HSD3B1(1245) genotype (SI Appendix, Fig. S3), and there is no association between genotype and circulating testosterone (SI Appendix, Fig. S4). Overall, daily GC therapy was associated with a $70 \%$ decrease in DHEA-S compared with no GC use in SARPIII and SARPI/II $(P<0.001)$ (SI Appendix, Table S3). Circulating DHEA also decreased by about $70 \%$ with GC therapy (SI $A p$ pendix, Fig. S5).

HSD3B1(1245) Genotype and GC Resistance. To test our hypothesis that the $H S D 3 B 1(1245 \mathrm{~A})$ adrenal restrictive allele is specifically associated with impaired lung function with GC treatmentmediated adrenal suppression, lung function was compared in $318 \mathrm{GC}$ and noGC Caucasian patients enrolled in SARP III for whom HSD3B1(1245) genotype data were available. Statistical comparisons of GC and noGC $\mathrm{FEV}_{1} \mathrm{PP}$ by HSD3B1(1245) genotype, before and after bronchodilation (BD), are summarized

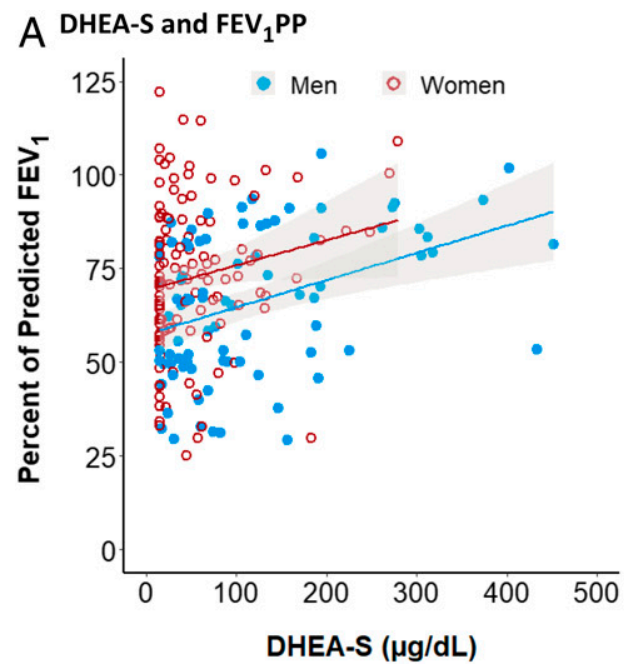

\section{B GC Use and DHEA-S}

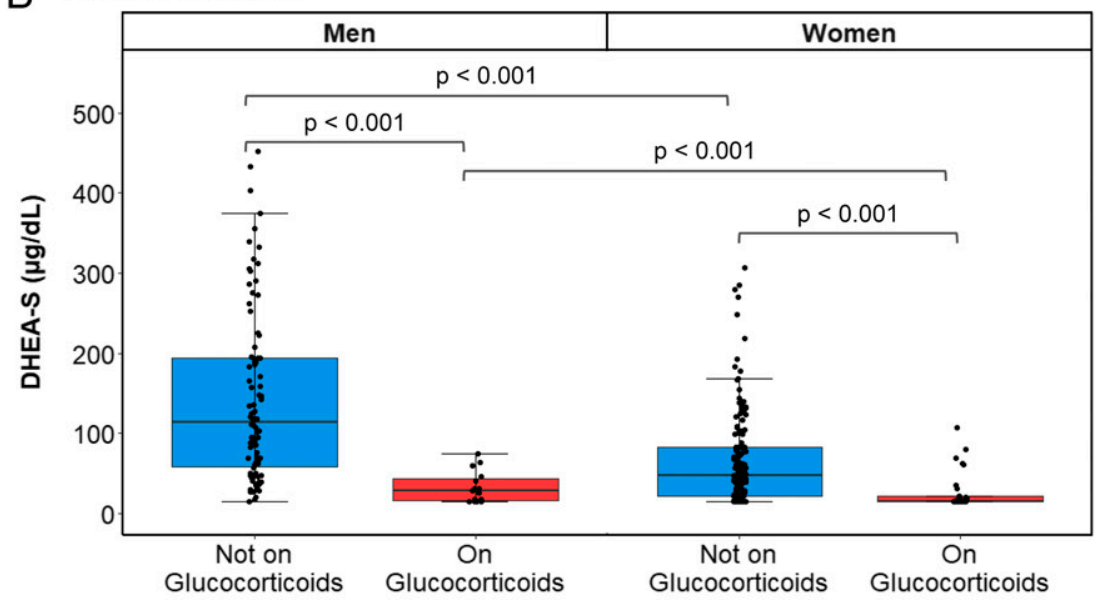

Fig. 1. DHEA-S concentration is associated with FEV ${ }_{1} \mathrm{PP}$ and suppressed with daily systemic GC use. $(A)$ In subjects with asthma, FEV ${ }_{1}$ PP correlates significantly with DHEA-S levels in both Caucasian men (blue circles; $R^{2}=0.15 ; P<0.001$ ) and women (open red circles; $R^{2}=0.03 ; P=0.047$ ). ( $B$ ) Chronic daily GC use is associated with DHEA-S suppression in SARP III in men and women. 
in Table 2, and graphical representations are presented in Fig. 2 $A$ and $B$. Overall, pre- or post-FEV ${ }_{1} \mathrm{PP}$ was not correlated with $H S D 3 B 1(1245)$ genotype in the noGC group. However, an interaction was present between daily GC use and HSD3B1(1245) genotype, suggesting that the effect of $H S D 3 B 1(1245)$ genotype depends on daily GC use. In patients with asthma homozygous for the HSD3B1(1245A) adrenal restrictive allele, pre-BD$\mathrm{FEV}_{1} \mathrm{PP}$ (Fig. $2 A$ ) for GC-treated individuals is significantly worse than for noGC $(54.3$ vs. $75.1 ; P<0.001)$. Post-BD$\mathrm{FEV}_{1} \mathrm{PP}$ for $\mathrm{GC}$-treated individuals with the homozygous adrenal restrictive genotype (Fig. $2 B$ ) is similarly worse than noGC (64.2 vs. 83.9; $P<0.001)$. In sharp contrast, for patients with asthma homozygous for the HSD3B1(1245C) adrenal permissive allele, the GC and noGC groups do not differ significantly for either pre-BD-FEV ${ }_{1} \mathrm{PP}(73.4$ vs. $78.9 ; P=0.41)$ or post-BD $\mathrm{FEV}_{1} \mathrm{PP}(83.1$ vs. $89.2 ; P=0.32)$. For patients with asthma who inherit the heterozygous genotype, the association of poorer outcomes in GC vs. noGC groups, although smaller in effect compared with the homozygous adrenal restrictive genotype, persists for pre-BD-FEV $\mathrm{PP}_{1}(59.4$ vs. 75.6; $P=0.001)$ and postBD-FEV ${ }_{1} \mathrm{PP}(67.7$ vs. $83.2 ; P<0.001)$. This relationship also holds when the results were stratified by sex (SI Appendix, Fig. S6). Results from the asthma control test similarly show that outcomes in the GC group are worse for patients with asthma who inherit at least one copy of the adrenal restrictive HSD3B1(1245A) allele (SI Appendix, Fig. S7), and that neutrophil counts significantly decrease in those who do not inherit the adrenal restrictive allele (SI Appendix, Fig. S8).

To test the association between the HSD3B1 adrenal restrictive allele and lower $\mathrm{FEV}_{1} \mathrm{PP}$ with oral GC treatment in a different cohort, we assessed 184 Caucasian patients with severe asthma in SARP I\&II. Mirroring the results in SARP III, patients homozygous for the $H S D 3 B 1(1245 \mathrm{~A})$ adrenal restrictive genotype in the GC group have significantly worse pre-BD$\mathrm{FEV}_{1} \mathrm{PP}(49.8$ vs. $63.4 ; P<0.001)$ and post-BD-FEV ${ }_{1} \mathrm{PP}(63.7$ vs. 73.5; $P<0.05$ ) than noGC (Fig. $2 C$ and $D$ ). Patients homozygous for the HSD3B1(1245C) adrenal permissive genotype have pre-BD-FEV ${ }_{1} \mathrm{PP}$ and post-BD-FEV ${ }_{1} \mathrm{PP}$ that are statistically indistinguishable between $\mathrm{GC}$ and noGC groups, effectively replicating the findings in SARP III. $\mathrm{FEV}_{1} \mathrm{PP}$ for heterozygote patients are again intermediate between AA (adrenal restrictive) and CC (adrenal permissive) genotypes. Additional statistical comparisons for SARP III and SARP I/II are shown in SI Appendix, Fig. S9. We acknowledge that sample size is a limitation of our study.
The mechanisms by which androgens can benefit asthma have been described in detail in animal models. In particular, androgens directly inhibit airway inflammation $(4,8,9,15)$, airway smooth muscle, and fibroblast proliferation (4-7). Consistent with these data, we have recently shown that DHEA therapy improves lung function in low-DHEA-S women (34). Moreover, our additional analysis shows that all women in our study whose serum DHEA-S increased by more than $300 \mu \mathrm{g} / \mathrm{dL}$ had an increase in FEV1 $(n=8)$ compared with those who had less of an increase in DHEA-S $(P=0.018$ by Fisher's exact test). Furthermore, the plausibility of DHEA/DHEA-S benefit in asthma is further supported by $3 \beta$-HSD1 expression in the human lung (SI Appendix, Fig. S10). A model that summarizes the effect of the association between HSD3B1(1245) genotype, DHEA-S suppression with GC treatment and $\mathrm{FEV}_{1} \mathrm{PP}$ is shown in Fig. 3.

\section{Discussion}

Severe asthma is defined as asthma that remains symptomatic and exacerbation-prone despite controlled high-dose inhaled ICS or systemic steroid treatment in conjunction with a second controller medication (3). Causes underlying severe asthma are heterogeneous $(28,30,35,36)$, and many patients are refractory, even to recently developed biological therapies (36). An aspect of severe asthma that is not commonly considered is that systemic GC therapy increases risk for low circulating levels of androgens, particularly DHEA-S (37).

Our study supports a model in which HSD3B1(1245) genotypes that confer less active conversion from adrenal precursors to potent androgens in peripheral tissues leads to a physiologic state of relative androgen deficiency that occurs specifically with DHEA-S suppression that is a consequence of systemic GC treatment. Strikingly, an HSD3B1(1245) allele-dose dependent effect appears to be clear and occurs for pre-BD-FEV ${ }_{1} \mathrm{PP}$ and post-BD-FEV ${ }_{1}$ PP in both SARP III and SARP I\&II.

In general, androgens require the AR to mediate much of their physiologic effects. Potent AR stimulation from adrenal DHEA/ DHEA-S, which is available in circulation, requires enzymatic conversion by $3 \beta$-HSD1 to testosterone and dihydrotestosterone, which occurs in peripheral tissues. Given the variety of tissues in which AR is expressed, the association observed in our study may be attributable to androgen stimulation in several different cell types. Androgens have many effects that could be beneficial for the asthmatic airway. For example, DHEA-S inhibits human airway smooth muscle and fibroblast proliferation and may benefit airway epithelial to mesenchymal transition (4-6). Both DHEA-S

Table 2. Comparison of maximum post-BD-FEV $\%$ between patients treated with and without daily oral GCs among the HSD3B1(1245) AA, AC, and CC genotypes*

\begin{tabular}{|c|c|c|c|c|c|c|}
\hline \multirow[b]{2}{*}{ HSD3B1(1245) Genotype } & \multicolumn{3}{|c|}{ SARP III } & \multicolumn{3}{|c|}{ SARP I \& II } \\
\hline & Not on GC & On GC & $P$ value & Not on GC & On GC & $P$ value $^{\dagger}$ \\
\hline \multicolumn{7}{|l|}{ AA } \\
\hline$n$ & 123 & 23 & & 45 & 35 & \\
\hline Pre-FEV1, $\%$ of predicted value & $75.1 \pm 18.3$ & $54.3 \pm 15.3$ & $<0.001$ & $63.4 \pm 18.8$ & $49.8 \pm 17.1$ & 0.001 \\
\hline Post-FEV1, $\%$ of predicted value & $83.9 \pm 18.8$ & $64.2 \pm 19.6$ & $<0.001$ & $73.5 \pm 15.9$ & $63.7 \pm 18.4$ & 0.024 \\
\hline \multicolumn{7}{|l|}{$A C$} \\
\hline$n$ & 107 & 24 & & 49 & 30 & \\
\hline Pre-FEV1, $\%$ of predicted value & $75.6 \pm 21.2$ & $59.4 \pm 19.8$ & 0.001 & $66.9 \pm 22.3$ & $54.3 \pm 18.3$ & 0.008 \\
\hline Post-FEV1, $\%$ of predicted value & $83.2 \pm 20.5$ & $67.7 \pm 17.9$ & $<0.001$ & $77.2 \pm 20.8$ & $67.8 \pm 20.3$ & 0.07 \\
\hline \multicolumn{7}{|l|}{ CC } \\
\hline$n$ & 33 & 8 & & 15 & 10 & \\
\hline Pre-FEV1, $\%$ of predicted value & $78.9 \pm 16.3$ & $73.4 \pm 16.6$ & 0.41 & $67.7 \pm 22.5$ & $66.7 \pm 26.6$ & 0.92 \\
\hline Post-FEV1, $\%$ of predicted value & $89.2 \pm 17.0$ & $83.1 \pm 14.4$ & 0.32 & $78.2 \pm 22.3$ & $80.7 \pm 16.2$ & 0.76 \\
\hline
\end{tabular}

*Data are presented as mean \pm SD for continuous variables and proportions or percentages for categorical variables.

${ }^{\dagger} P$ value comparing daily oral GC vs. no GC in each genotype group $A A, A C$, and $C C$. 

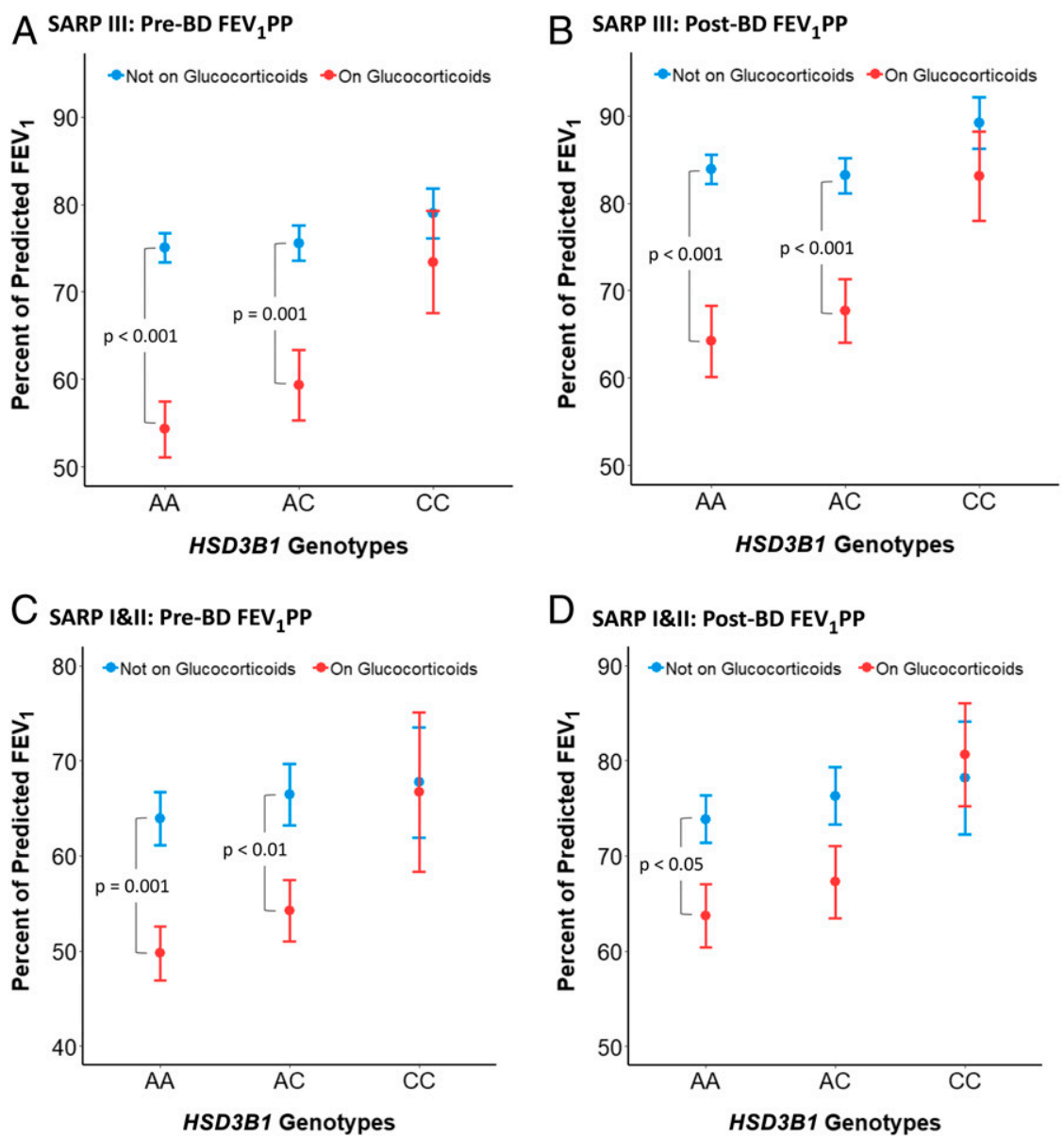

Fig. 2. The adrenal restrictive $H S D 3 B 1(1245 \mathrm{~A})$ allele is specifically associated with poor pulmonary function in GC-treated patients with severe asthma. In Caucasian AA genotype patients with asthma enrolled in SARP III, baseline prebronchodilator FEV ${ }_{1}$ PP (Pre-BD FEV ${ }_{1}$ PP) $(A)$ and postbronchodilator FEV ${ }_{1}$ PP (Post-BD FEV 1 PP) $(B)$ is lower for those in the GC vs. no GC treatment groups. In contrast, for the CC genotype, there is no difference between GC and no GC treatment groups. Lower Pre-BD FEV 1 PP $(C)$ and Post-BD FEV ${ }_{1} P P(D)$ for AA genotype patients receiving GC also occurs in Caucasian patients with severe asthma enrolled in SARP I\&II. Error bars indicate SEs.

and testosterone promote airway smooth muscle relaxation (7). Testosterone is associated with decreased Th2 and Th1 inflammation in animal models of asthma $(8,9)$. Epidemiologically, androgens are associated with better lung function in large healthy cohorts $(10,11)$ and in disease (12). Increasing circulating levels of adrenal and gonadal androgens in males and females during adolescence are believed to be associated with improving asthma during adolescence (12), and gonadal androgens in particular may be associated with gender-based differences in asthma incidence and severity in adulthood $(12,38-40)$. Notably, steroid metabolites downstream of DHEA-S are generated at the level of the target peripheral tissue and are generally not appreciable in circulation $(41,42)$.

These data suggest that androgen depletion, whether circulating and/or at the tissue level, could contribute to the pathophysiology

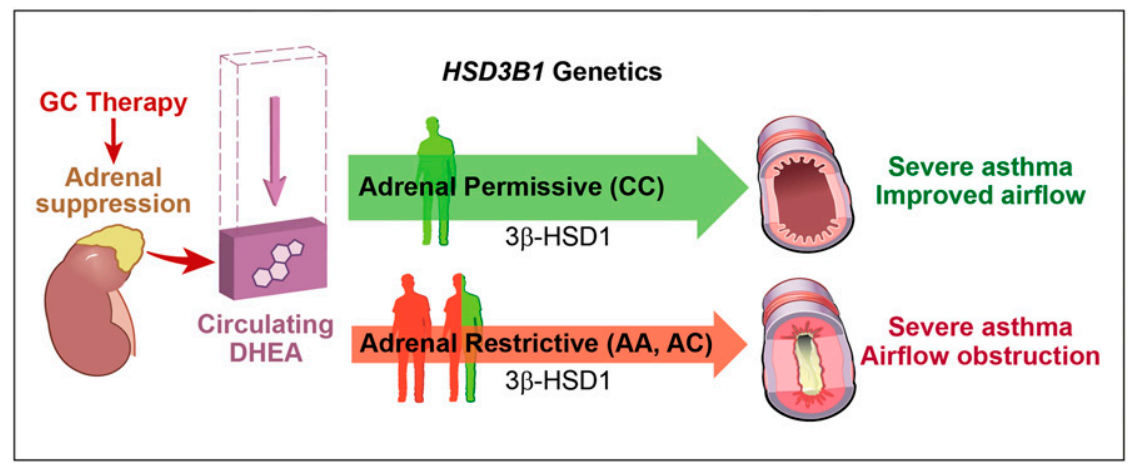

Fig. 3. A model that explains physiologic effects of $H S D 3 B 1$ inheritance on $F E V_{1} P P$ in patients with severe asthma. GC treatment suppresses adrenal DHEA, which may become a limiting substrate for 3 3 -HSD1, depending on HSD3B1 genotype. Adrenal permissive and adrenal restrictive alleles enable and limit metabolic flux through 3 $\beta$-HSD1 and recovery of airflow. 
of severe asthma and resistance to oral GC therapy. Our data confirm that low DHEA-S levels in asthma are associated with low lung function. However, we do not know whether this is a causal relationship, whether low androgen levels are simply a marker associated with patients with low lung function on more GCs, or both. We recently published a pilot study suggests that DHEA supplementation in women with low DHEA-S improves $\mathrm{FEV}_{1}$ (34).

Recent studies of prostate cancer have shown that the adrenal restrictive $H S D 3 B 1(1245 \mathrm{~A})$ allele limits conversion of DHEA to more potent androgens, whereas the adrenal permissive $H S D 3 B 1(1245 \mathrm{C})$ allele increases tissue production of potent androgens $(16,17)$. The SARP studies provided an opportunity to study androgen metabolism effects in severe asthma. We therefore hypothesized that the restrictive allele, which impedes conversion from adrenal DHEA-S to testosterone and dihydrotestosterone, would be associated with lower lung function in severe asthma when systemic GCs are used and suppress substrate availability for $3 \beta$ HSD1. Data were analyzed in the SARP III cohort, enriched for severe asthma, and then validated in the patients with severe asthma in the SARP I\&II cohort and are strikingly consistent with our hypothesis across both cohorts.

Limitations of this study include that it is restricted to Caucasian patients with asthma and the sample size, particularly for patients with adrenal permissive HSD3B1(1245C) genotypes. The low frequency of the adrenal permissive $H S D 3 B 1(1245 \mathrm{C})$ allele in African-American subjects combined with the limited number of subjects did not allow for a sufficiently powered analysis in nonCaucasians. For example, only 1 African American participant with CC genotype was enrolled in SARP III, and 2 others in SARP I\&II. The lower prevalence of the adrenal permissive C allele in African Americans could contribute to higher severity and risk from asthma, as well as GC resistance, in this patient population. Similarly, none of the 27 adult Hispanic participants with asthma enrolled in SARP III, and only 1 of the 34 adult Hispanics enrolled in SARP I\&II, respectively, carried the CC genotype. Of note, the adrenal permissive allele frequency (i.e., the $\mathrm{C}$ allele) was $36 \%$ and $37 \%$ in adult Caucasian participants

1. P. S. Hench, C. H. Slocumb, H. F. Polley, E. C. Kendal, Effect of cortisone and pituitary adrenocorticotropic hormone (ACTH) on rheumatic diseases. J. Am. Med. Assoc. 144, 1327-1335 (1950).

2. P. J. Barnes, I. M. Adcock, Glucocorticoid resistance in inflammatory diseases. Lancet 373, 1905-1917 (2009)

3. K. F. Chung et al., International ERS/ATS guidelines on definition, evaluation and treatment of severe asthma. Eur. Respir. J. 43, 343-373 (2014) Erratum in: Eur Respir J. 43, 1216 (2014).

4. C. J. Koziol-White et al., DHEA-S inhibits human neutrophil and human airway smooth muscle migration. Biochim. Biophys. Acta 1822, 1638-1642 (2012).

5. C. Mendoza-Milla et al., Dehydroepiandrosterone has strong antifibrotic effects and is decreased in idiopathic pulmonary fibrosis. Eur. Respir. J. 42, 1309-1321 (2013).

6. L. Xu et al., Effects and mechanism of dehydroepiandrosterone on epithelialmesenchymal transition in bronchial epithelial cells. Exp. Lung Res. 40, 211-221 (2014).

7. V. Kouloumenta, A. Hatziefthimiou, E. Paraskeva, K. Gourgoulianis, P. A. Molyvdas, Non-genomic effect of testosterone on airway smooth muscle. Br. J. Pharmacol. 149, 1083-1091 (2006).

8. J. Y. Cephus et al., Testosterone attenuates group 2 innate lymphoid cell-mediated airway inflammation. Cell Rep. 21, 2487-2499 (2017).

9. H. Fuseini et al., Testosterone decreases house dust mite-induced type 2 and IL-17amediated airway inflammation. J. Immunol. 201, 1843-1854 (2018).

10. J. Svartberg, H. Schirmer, A. Medbø, H. Melbye, U. Aasebø, Reduced pulmonary function is associated with lower levels of endogenous total and free testosterone. The Tromsø study. Eur. J. Epidemiol. 22, 107-112 (2007).

11. S. S. Mohan et al., Higher serum testosterone and dihydrotestosterone, but not oestradiol, are independently associated with favourable indices of lung function in community-dwelling men. Clin. Endocrinol. (Oxf.) 83, 268-276 (2015).

12. M. D. DeBoer et al., Effects of endogenous sex hormones on lung function and symptom control in adolescents with asthma. BMC Pulm. Med. 18, 58 (2018).

13. R. J. Auchus, The physiology and biochemistry of adrenarche. Endocr. Dev. 20, 20-27 (2011).

14. N. Sharifi, Minireview: Androgen metabolism in castration-resistant prostate cancer. Mol. Endocrinol. 27, 708-714 (2013)

15. J. Hazeldine, W. Arlt, J. M. Lord, Dehydroepiandrosterone as a regulator of immune cell function. J. Steroid Biochem. Mol. Biol. 120, 127-136 (2010). with asthma enrolled in SARP III and severe asthma enrolled SARP I\&II. Those frequencies match the frequency reported for Caucasians in the 1000 Genomes Project and suggest the absence of any deviation from Hardy-Weinberg Equilibrium.

In conclusion, we have identified a genetic determinant associated with GC resistance and low lung function in asthma. Evidence that the adrenal restrictive HSD3B1(1245A) allele, which confers a lower level of prostate cancer adrenal androgen dependence by preventing local conversion of DHEA to more potent androgens, adversely affects lung function in GC-dependent severe asthma suggests that androgens have a central role in the pathophysiology of human severe asthma and response to systemic GC treatment. Our data provide genetic evidence that complements epidemiological data regarding a benefit of androgens on lung function. Our model is further supported by our data showing a positive relationship between circulating adrenal DHEA-S levels and lung function. Indeed, androgens cause airway smooth muscle relaxation and prevent both remodeling and inflammation in animal models of asthma. GC use suppresses endogenous androgen production, preventing the beneficial effects of androgens in human asthma. These data suggest the possibility that $H S D 3 B 1$ genotype is predictive of which patients might benefit from systemic GC therapy alone and, for those who are resistant, who would benefit from androgen replacement in severe asthma.

ACKNOWLEDGMENTS. We thank the patients and their families for their participation in the Severe Asthma Research Program; the site-based study coordinators and regulatory personnel for enrolling participants, the Data Safety and Monitoring Board for program oversight, and National Institute of Health/National Heart, Lung, and Blood Institute project officers and staff who administered the protocol. Supported by a grant from the National Heart, Lung, and Blood Institute Severe Asthma Research Program (U10 HL109250, P01 HL128192, and P01 HL101871 to Rainbow Babies and Children's Hospital Virginia-Cleveland Consortium, and RO1 HL69170, U10 HL109250, K08 HL133381, P01 HL103453, P01 HL081064, R01CA172382, R01CA190289, R01 CA236780, and a grant from the Prostate Cancer Foundation to the Cleveland Clinic). We also thank the Harrington Discovery Institute of University Hospitals, Cleveland, for grant support.

16. K. H. Chang et al., A gain-of-function mutation in DHT synthesis in castration-resistant prostate cancer. Cell 154, 1074-1084 (2013).

17. J. W. D. Hearn et al., HSD3B1 and resistance to androgen-deprivation therapy in prostate cancer: A retrospective, multicohort study. Lancet Oncol. 17, 1435-1444 (2016).

18. N. Agarwal et al., Independent validation of effect of HSD3B1 genotype on response to androgen-deprivation therapy in prostate cancer. JAMA Oncol. 3, 856-857 (2017).

19. J. W. D. Hearn et al., Association of HSD3B1 genotype with response to androgendeprivation therapy for biochemical recurrence after radiotherapy for localized prostate cancer. JAMA Oncol. 4, 558-562 (2018).

20. M. Shiota et al., Association of missense polymorphism in HSD3B1 with outcomes among men with prostate cancer treated with androgen-deprivation therapy or abiraterone. JAMA Netw. Open 2, e190115 (2019).

21. S. R. R. Garcia Gil et al., Relationship between Mutations in the HSD3B1 Gene and Response Time to Androgen Deprivation Therapy in the Treatment of Prostate Cancer (European Society of Oncology Pharmacy, Nantes, France, 2018).

22. J. W. D. Hearn et al., HSD3B1 genotype and clinical outcomes in metastatic castrationsensitive prostate cancer. JAMA Oncol., in press.

23. V. Brusasco, R. Crapo, G. Viegi; American Thoracic Society; European Respiratory Society, Coming together: The ATS/ERS consensus on clinical pulmonary function testing. Eur. Respir. J. 26, 1-2 (2005).

24. M. R. Miller et al.; ATS/ERS Task Force, Standardisation of spirometry. Eur. Respir. J. 26 319-338 (2005).

25. P. H. Quanjer et al.; ERS Global Lung Function Initiative, Multi-ethnic reference values for spirometry for the 3-95-yr age range: The global lung function 2012 equations. Eur. Respir. J. 40, 1324-1343 (2012).

26. W. G. Teague, B. R. Phillips, J. V. Fahy et al., Baseline features of the severe asthma research program (SARP III) cohort: Differences with age. J. Allergy Clin. Immunol. Pract. 6, 545-554 (2018)

27. W. Phipatanakul et al.; Severe Asthma Research Program, Effects of age and disease severity on systemic corticosteroid responses in asthma. Am. J. Respir. Crit. Care Med. 195, 1439-1448 (2017)

28. W. C. Moore et al.; National Heart, Lung, Blood Institute's Severe Asthma Research Program, Characterization of the severe asthma phenotype by the national Heart, lung, and blood institute's severe asthma research program. J. Allergy Clin. Immunol. 119, 405-413 (2007) 
29. N. N. Jarjour et al.; NHLBI Severe Asthma Research Program (SARP), Severe asthma: Lessons learned from the national Heart, lung, and blood Institute severe asthma research program. Am. J. Respir. Crit. Care Med. 185, 356-362 (2012).

30. W. C. Moore et al.; National Heart, Lung, and Blood Institute's Severe Asthma Research Program, Identification of asthma phenotypes using cluster analysis in the Severe Asthma Research Program. Am. J. Respir. Crit. Care Med. 181, 315-323 (2010).

31. American Thoracic Society, Proceedings of the ATS workshop on refractory asthma: Current understanding, recommendations, and unanswered questions. Am. J. Respir. Crit. Care Med. 162, 2341-2351 (2000).

32. S. Purcell et al., PLINK: A tool set for whole-genome association and population-based linkage analyses. Am. J. Hum. Genet. 81, 559-575 (2007).

33. C. C. Chang et al., Second-generation PLINK: Rising to the challenge of larger and richer datasets. Gigascience 4, 7 (2015).

34. N. Marozkina et al., Dehydroepiandrosterone supplementation may benefit women with asthma who have low androgen levels: A pilot study. Pulm. Ther. 5, 213-220 (2019)

35. The ENFUMOSA cross-sectional European multicentre study of the clinical phenotype of chronic severe asthma. European network for understanding mechanisms of severe asthma Eur. Respir. J. 22, 470-477 (2003).
36. L. C. Denlinger et al., Inflammatory and Co-morbid features of patients with severe asthma and frequent exacerbations. Am. J. Respir. Crit. Care Med. 195, 302-313 (2017). Erratum in: Am. J. Respir. Crit. Care Med. 197, 971 (2018).

37. M. J. Dorsey, L. E. Cohen, W. Phipatanakul, D. Denufrio, L. C. Schneider, Assessment of adrenal suppression in children with asthma treated with inhaled corticosteroids: Use of dehydroepiandrosterone sulfate as a screening test. Ann. Allergy Asthma Immunol. 97, 182-186 (2006).

38. K. G. Tantisira et al.; Childhood Asthma Management Program Research Group, Airway responsiveness in mild to moderate childhood asthma: Sex influences on the natural history. Am. J. Respir. Crit. Care Med. 178, 325-331 (2008).

39. M. R. Becklake, F. Kauffmann, Gender differences in airway behaviour over the human life span. Thorax 54, 1119-1138 (1999).

40. S. M. Tse et al., Sex-specific risk factors for childhood wheeze and longitudinal phenotypes of wheeze. J. Allergy Clin. Immunol. 138, 1561-1568 (2016).

41. F. Labrie, Extragonadal synthesis of sex steroids: Intracrinology. Ann. Endocrinol. (Paris) 64, 95-107 (2003)

42. N. Sabharwal, N. Sharifi, HSD3B1 genotypes conferring adrenal-restrictive and adrenal-permissive phenotypes in prostate cancer and beyond. Endocrinology 160, 2180-2188. 